\title{
INVERSIÓN EN INTANGIBLES Y ESTRATEGIA COMPETITIVA: UNA EXTENSIÓN DEL MODELO DE COURNOT
}

\author{
Gloria Inés Sarmiento* \\ Óscar Arturo Benavides**
}

T a inversión -la adquisición o creación de recursos para usarlos en la producción- "se concentra en la producción o adquisición de capital físico, construcciones, equipos e inventarios", aunque también "incluye la adquisición de capital humano e intangible" (Coen y Eisner, 1987, 980). No obstante, desde hace tiempo la literatura reconoce la importancia de algunos elementos intangibles en la producción. Marshall (citado por Coase, 1937, 388) considera que el capital organizacional es un factor productivo; Veblen $(1908,124)$ admite que los “activos intangibles son capital así como los activos tangibles". En la literatura reciente sobre organización industrial ${ }^{1}, \mathrm{y}$ desde finales de los años ochenta en los modelos de crecimiento endógeno ${ }^{2}$, se resalta la importancia de la inversión en intangibles para las firmas y para toda la economía.

La importancia de la inversión en activos intangibles también se destaca en estudios empíricos. El Banco Mundial (2006) y la OCDE (2012) muestran su relevancia a nivel agregado. El trabajo del Banco estudia 120 países y analiza la composición de su riqueza ${ }^{3}$. Los resultados muestran que la riqueza de los países de bajo ingreso equivale

* Magíster en Economía y M. A. en Política Económica, economista senior del Banco de la República, [gsarmibe@banrep.gov.co].

** Doctor en Ciencias Económicas, profesor asociado de la Universidad Nacional de Colombia, Bogotá, [oabenavidesg@unal.edu.co]. Fecha de recepción: 27-09-2016, fecha de modificación: 22-08-2017, fecha de aceptación: 04-09-2017. Sugerencia de citación: Sarmiento, G. I. y Benavides, Ó. A. (2017). Inversión en intangibles y estrategia competitiva: una extensión del modelo de Cournot, Revista de Economía Institucional 19(37), 85-114. DoI: https://doi.org/10.18601/01245996.v19n37.05

${ }^{1}$ Andonova y Ruiz (2016). Golec y Gupta (2014), Tsai y Yen (2012) y Sutton (1996).

${ }^{2}$ Romer (1990) y Aghion y Howitt (1990).

${ }^{3}$ Aunque en este trabajo se cuantifica el capital intangible de toda la economía y no solo el de las firmas, la definición cambia un poco (p. ej., no se considera 
al $2 \%$ de la de los de alto ingreso y que su composición es diferente. Mientras que la participación del capital físico es relativamente estable (entre el 16\% y el 19\%), la del capital intangible, que allí incluye el capital social, las instituciones y el capital humano, es mayor en los países de alto ingreso (80\%) que en los de ingreso medio (68\%) y bajo (59\%), y que la participación del capital natural (recursos naturales) es mayor en los países de ingreso bajo (26\%) que en los de ingreso medio y alto ( $13 \%$ y $2 \%$ respectivamente $)^{4}$.

A nivel de las firmas, Brynjolfsson et al. (2002) presentan varios casos en los que resaltan la importancia de los intangibles. Dell Computer, por ejemplo, mediante una combinación de software y rediseño del flujo de tareas redujo el área de ensamblaje a casi la mitad y el inventario de productos en proceso; así duplicó su capacidad productiva. Sin esos cambios habría tenido que construir una planta del mismo tamaño de la original. Johnson \& Johnson también disminuyó en forma significativa los costos de producción de bandas adhesivas combinando nueva maquinaria computarizada y "una decena de prácticas y principios cuidadosamente definidos" (Brynjolfsson et al., $2002,147)$ que incluían cambios en la toma de decisiones y sistemas de incentivos y responsabilidades. Uchitelle (2006) resalta el caso de Walmart, que diseñó un sistema eficiente de inventarios. Andonova y Ruiz (2016) muestran que la inversión en activos intangibles juega un papel muy importante en el desempeño de las firmas en Colombia entre 1995-2012.

Hennings $(1987,329)$ argumenta, citando a Maitland (1804) que "lo que puede remplazar a un factor de producción se debe considerar de la misma especie". Varios autores ${ }^{5}$, el Banco Mundial, la ocDE, el Bureau of Economic Analysis de Estados Unidos y el Central Bureau of Statistics de Holanda reconocen que elementos intangibles como la investigación y el desarrollo (I\&D), la publicidad y la capacitación se deben considerar como inversión y no como gasto. Así, Mankiw sostiene que "la máquina de coser automática [...] se puede contabilizar en el PIв como inversión porque, una vez instalada, hace un vestido tras otro, y genera una corriente de ingresos. De modo similar,

el capital relacional ni la reputación): es el de mayor cobertura y permite hacer comparaciones entre países porque aplica la misma metodología para todos.

${ }^{4} \mathrm{La}$ ocDe señala que un $50 \%$ del valor de las firmas es atribuible a los activos intangibles. Chun et al. (2015) muestran la importancia de la inversión en intangibles en el aumento de la productividad en Japón y Corea, y consideran necesario crear mercados financieros que permitan valorar en forma explícita el capital intangible.

${ }_{5}^{5}$ Como David (2004), Mankiw (2006) y McGrattan y Prescott (2006). 
la I\&D que hizo el Prozac quizá produzca ingresos por años, así como la máquina de coser" 6 .

Según la literatura, la inversión en bienes intangibles es al menos un $30 \%$ de la inversión en capital físico y equivale a varios puntos del рів. Según el Bureau of Economic Analysis, las firmas realizan entre un $50 \%$ y un $70 \%$ de la inversión en $\mathrm{I}_{2} \mathrm{D}^{7}$. Cabe entonces preguntar: ¿por qué la sociedad y las firmas invierten en intangibles? La respuesta usual sería que, entre las alternativas de inversión, la firma elige la que produce un rendimiento marginal mayor $y$, si invierte en capital intangible, la razón es que el aumento de su acervo produce un rendimiento mayor que el aumento del capital físico.

Para Porter la respuesta es que las firmas invierten para mantener una ventaja competitiva, lo cual logran cuando dan "a los consumidores lo que quieren o necesitan, de una manera mejor y más efectiva que los competidores, y que es difícil de imitar" (Jonhson y Scholes, 2001, 244). Igual que los economistas, los administradores piensan que la firma busca maximizar los beneficios, pero reconocen que intenta limitar la competencia, porque reduce el rendimiento al mínimo cuando la firma no tiene ventaja competitiva: en competencia perfecta (Porter, 1986). La estrategia competitiva combina los mecanismos que la firma emplea para reducir la competencia.

En esta idea concordarían autores como Webster (1999, 2002 y 2006), Lorente (2000) y Andonova y Ruiz (2016), para quienes los intangibles son una fuente de ventaja competitiva sostenible para las firmas. La literatura teórica sobre el capital intangible es incipiente y la mayoría es de carácter empírico. Los trabajos teóricos se concentran en definirlo y ofrecer guías para medirlo. Desde luego, los trabajos empíricos parten de una teoría implícita o explícita del capital intangible o de la inversión en intangibles, aun aquellos que solo intentan cuantificar su valor, pero no analizan cómo se produce, cuáles son los incentivos de la firma ni por qué obtiene beneficios superiores a los de la industria y una posición dominante.

Este artículo busca mostrar, mediante un modelo tipo Cournot, cómo se producen los intangibles en las firmas y sus incentivos para realizar estas inversiones. E1 modelo, de bienes homogéneos y competencia simultánea, y cantidades como variable estratégica, es adecuado porque proporciona resultados para diferentes estructuras de mercado -monopolio, oligopolio y competencia perfecta- antes y después de la inversión en intangibles. Aquí no se analiza la competencia mo-

\footnotetext{
${ }^{6}$ Ver [http://gregmankiw.blogspot.com/2006_04_01_archive.html].

7 Ver las cifras en [http://www.bea.gov/rd/xls/1959_2004_rd_data.xls].
} 
nopolística, pero cabe señalar que la diferenciación de bienes es una estrategia competitiva, el resultado de invertir en intangibles como publicidad y mercadeo. Solo considera las inversiones que reducen costos para mostrar hasta qué punto hacen posible obtener beneficios superiores a los de sus competidores. El modelo parte de la principal característica del capital intangible: no se produce en forma masiva y la firma debe producirlo por sí misma. Por ello es difícilmente replicable y, según Porter, las firmas deben encontrar maneras de producirlo que sean difíciles de reproducir para tener una ventaja competitiva. La primera parte del artículo muestra qué es el capital intangible, cuáles son sus características y por qué su adquisición se debe considerar como inversión; en la segunda se presenta el modelo y en la última, las conclusiones.

\section{LA INVERSIÓN EN INTANGIBLES}

\section{LA INVERSIÓN Y EL CAPITAL}

Primero es conveniente definir qué es inversión. Según la definición más común es "formación de capital" (Coen y Eisner, 1987, 980), y el capital consiste en los recursos utilizados en la producción, que intervienen en varios ciclos productivos. Es entonces un acervo de "medios de producción producidos" cuyo "valor no proviene del trabajo y la tierra utilizados en el pasado para fabricarlos [...] sino de que son útiles en la futura elaboración de productos" (Hennings, 1987, 330). Así, el capital es un conjunto de factores de producción durables que no solo incluye capital físico (planta, equipo, inventarios) sino también capital humano, de modo que los recursos dedicados a educación, salud, I\&D y capacitación de trabajadores son inversión (Sachs y Larrain, 1994; Coen y Eisner, 1987; Caballero, 1998).

La inversión aumenta, o al menos conserva, la capacidad productiva, e invertir significa "transar el presente por el futuro" (Caballero, $1998,815)$, de modo que el valor del producto generado por el capital adicional debe ser mayor que el de los recursos utilizados para crearlo (Coen y Eisner, 1987, 981). Las firmas realizan la mayoría de la inversión porque tienen a su cargo el proceso productivo, pero las familias y los gobiernos también invierten. En casi todos los países el gasto en educación e investigación se registra como consumo en las cuentas nacionales, lo que subestima la inversión y sobreestima el consumo (Sachs y Larrain, 1994, 118; Nakamura, 1999, 4).

Según Ducharme (1998, 3), el término "inversión en intangibles" se acuñó en los años ochenta para describir actividades que comple- 
mentaban o remplazaban la adquisición de capital físico, las cuales, según estudios empíricos, explican gran parte del aumento de la productividad. De ser así, el acervo que alimenta tales actividades es capital, como argumenta Hennings citando a Lauderdale. Webster $(2002,4)$ define el capital intangible como "toda forma de capital no incorporado en materia", y deduce que es un acervo que contribuye a generar producto en el futuro. Esta descripción recoge el consenso de la literatura. El cuadro 1 presenta algunas de las definiciones de activos intangibles e inversión en intangibles.

Cuadro 1

Definición de capital intangible

Autor $\quad$ Definición

OCDE plazo que realizan las firmas para mejorar su desempeño futuro, diferentes de la compra de activos fijos".

Los intangibles son "activos sin sustancia física, pero resultado de

Belkaoui (1992) derechos legales o contractuales, que probablemente produzcan beneficios futuros".

Los activos intangibles son "fuentes no físicas de posibles beneficios

Intangibles Research futuros para una firma, adquiridos por intercambio o desarrollados Center, New York Uni- internamente con costos identificables; tienen vida finita, valor de versity mercado independiente de la firma y son propiedad o controlados por ella".

Los activos intangibles son "recursos controlados por la firma, que

Arthur Andersen (1992) no son físicos por naturaleza, capaces de producir futuros beneficios netos, y protegidos por derechos legales o de hecho".

Los activos intangibles se definen como "activos sin sustancia física no monetarios, para usar en la producción u oferta de bienes o ser-

IASC 38 (1998) vicios, rentarlos a otros, o con propósitos administrativos". Deben ser a) identificables, b) controlados por la firma como resultado de eventos pasados y c) a partir de ellos se deben esperar beneficios futuros.

Vosselman (1998)

La inversión en intangibles "comprende los gastos de capital en productos intangibles disponibles en el periodo y que permanecen en uso por más de un año".

Fuente: Cañibano et al. (1999).

Existe consenso sobre las actividades que integran el acervo intangible que contribuye a generar ingresos futura netos a la firma, porque reducen costos o aumentan los ingresos. En general se considera inversión en intangibles los gastos en I\&D, capacitación, software y mercadeo, rubros que aparecen en la mayoría de los estudios empíricos (como los de la ocDE sobre diferentes países $)^{8}$ y en trabajos sobre inversión

${ }^{8}$ La ocde (1998) señala que, en el taller de 1992, seis de los países que presentaron informes (Finlandia, Noruega, Francia, Alemania, Suecia e Inglaterra) incluían I\&D, software y algunos aspectos del mercadeo; todos incluían capacitación, salvo Suecia. 
en intangibles (Young, 1999; Vosselman, 1999) ${ }^{9}$, aunque también mencionan actividades que constituyen acervos organizacionales de las firmas difíciles de identificar.

De esos elementos, la economía reconoce como fuentes importantes de crecimiento la I\&D y la capacitación. Para Shy $(1996,222)$, I\&D "es el acto de crear o cambiar la función de producción", y se traduce en nuevos productos o reducción de costos. En el primer caso, el aumento de la capacidad productiva parte de cero; en el segundo, con los mismos recursos se obtiene más producto. Sin embargo, se considera a la I\&D como un elemento ajeno de la función de producción más que como una actividad que incrementa un factor productivo, excepto en algunos modelos de crecimiento endógeno, como el de Romer (1990). En diversos trabajos la capacitación y la educación se consideran fuente de crecimiento económico y el capital humano como factor productivo. Ducharme (1998, 4-5), que cita a Becker (1975) y a Mincer (1989), sostiene que los recursos humanos son el factor productivo más importante y que el capital humano interviene en el proceso productivo como un factor que es fuente de innovación. Entre los modelos que resaltan la importancia del capital humano en el proceso productivo se encuentran los de Arrow (1962), Lucas (1988) y Romer $(1990)^{10}$. E1 software puede reducir costos directamente (como en el ejemplo anterior de Dell Computer), o reducir costos de transacción (p. ej., si al facilitar la comunicación disminuye el tiempo para tomar decisiones) y así aumentar la capacidad productiva (Vosselman, 1998).

La literatura económica considera el mercadeo y la publicidad más como medios para aumentar los beneficios de la firma que para incrementar el producto. Por ejemplo, Shy (1996) presenta modelos en los que la publicidad, las garantías y el soporte técnico afectan la demanda, y vía precios aumentan los beneficios. Como los intangibles no se pueden tocar, se asocian a servicios, y estos no se pueden almacenar, lo cual es una condición necesaria para ser un acervo (Vosselman, 1999) ${ }^{11}$. Sin embargo, la información que se almacena en un computador no tiene materia y constituye un cúmulo. Por tanto, es necesario identificar el tipo de acervo para que se pueda considerar

\footnotetext{
${ }^{9}$ En la literatura se encuentran argumentos en favor y en contra de su carácter de factor productivo. P. ej., que la I\&D modifica la función de producción pero no hace parte de ella, o que la publicidad solo hace creer a los consumidores que un bien es diferenciado (Shy, 1996, 282, citando a Kaldor).

${ }_{10}$ Estos modelos se presentan en Aghion y Howitt (1999).

11 Cuando se asocia el capital intangible a un servicio se considera más la inversión en capital intangible que el capital en sí mismo. P. ej., la publicidad o la capacitación se consideran servicios, pero no lo que queda después.
} 
capital intangible. Lorente (2000) da una pista al clasificar los intangibles en tres grupos: recursos reputacionales (publicidad), recursos tecnológicos (I\&D) y recursos humanos (capacitación) ${ }^{12}$.

Cabe señalar dónde se almacenan dichos acervos. El capital humano está incorporado en el trabajador; el capital organizacional, en elementos tales como el organigrama, el reglamento y los procedimientos formales o informales; el capital relacional o de mercado, en los consumidores y en la red de distribución; y el capital de producción, en recetas, instructivos y documentos legales como las patentes. Así, aunque la firma destine recursos a formar ese capital intangible, por depositarlo en el trabajador, en el consumidor o en prácticas formales o informales, quizá no tenga control total sobre ellos y, por tanto, no pueda apropiarse todo el rendimiento.

\section{¿POR QUÉ LOS INTANGIBLES SE DEBEN CONSIDERAR COMO CAPITAL?}

Como ya se mencionó, los pocos trabajos teóricos sobre capital intangible intentan definirlo y ofrecer guías para valorarlo y estimar la inversión en intangibles ${ }^{13}$. No obstante, sugieren otras razones, además de las ya señaladas, para considerar que el gasto en intangibles es inversión. Webster (2006) clasifica estos trabajos en tres grupos:

a. Tasa de retorno: su objetivo es calcular la tasa de retorno de la inversión en intangibles. Lorente (2000) et al., mediante un panel de firmas españolas, analizan el efecto de los recursos tecnológicos, humanos y reputacionales en la rentabilidad de la firma. Concluyen que los tres tienen efectos positivos si se utilizan medidas de rentabilidad más amplias que las contables. La principal crítica a este enfoque es que en la realidad no se dispone de una tasa de retorno que no incluya el capital intangible.

b. Función de producción: incluyen expresamente el capital intangible en la función de producción. Usando un modelo de crecimiento que involucra capital intangible, David (2004) muestra que las variaciones en las relaciones entre capital intangible, tangible y producto obedecen a cambios de precios relativos y que el descenso en la productividad en Estados Unidos desde 1960 se puede deber a una mala medición del producto.

${ }^{12}$ Según Johanson (1999), algunos autores usan la palabra recursos en vez de activos o capital porque es más amplia, pues involucra elementos que solo intervienen en un ciclo productivo (lo que no ocurre con el capital) y sobre los que la firma no tiene necesariamente el control (lo que no sucede con los activos). P. ej., una vez el trabajador adquiere los conocimientos que le brinda la firma a través de la capacitación, esta no controla lo que el empleado pueda hacer con ellos.

${ }^{13}$ Ver, p. ej., Young (1999), Vosselman (1999), Webster (2002), Cañibano et al. (1999) y Lev (2003). 
En un modelo de 2 sectores (bienes finales y capital intangible), McGrattan y Prescott (2006) conciben la inversión en intangibles como adquisiciones, o gastos contables de las firmas, y como horas extras de trabajo no remuneradas, que permiten a los empleados obtener ganancias futuras. Comparan los resultados del modelo acerca de la PTF con los datos observados en Estados Unidos y, como las trayectorias son cercanas, concluyen que las medidas estándar subestiman los aumentos de productividad.

O’Mahony y Vecchi (2002) hacen un análisis de clúster de firmas de varios países y encuentran que obtienen rendimientos crecientes a escala en virtud de la I\&D y que la productividad de las firmas de sectores intensivos en capital humano e I\&D es un 3,7\% más alta. Por último, Peneder (2002), también en un análisis de clúster, encuentra que las industrias difieren en su propensión a invertir en intangibles (I\&D, capital humano y publicidad) y que hay alta complementariedad entre capital humano y gastos en I\&D.

c. Valor de mercado: suponen que los mercados de capitales valoran correctamente el flujo futuro de beneficios de la firma y que su precio de mercado es igual al valor presente de los beneficios. Como se conoce el precio del capital físico ${ }^{14}$, la diferencia entre el valor de mercado de la firma y el del capital físico es el capital intangible. Webster (1999) encuentra entonces que entre 1947 y 1998 (excluyendo el periodo 1974-1984) la relación entre capital intangible y capital total creció un 1,25\% por año. Brynholfsson et al. (2002), con datos de 1.216 firmas de 1987 a 1997, encontraron que por cada dólar gastado en computadores (como medida de tecnologías de información) el precio de mercado de la firma aumentó en 10 dólares (la inversión en capital físico generaba un aumento proporcional) y que esas inversiones se correlacionaban con prácticas organizacionales observables (capacitación, formación de equipos, etc.) que quizá contribuían a ese aumento.

Con un modelo de costos de ajuste e información de firmas de Estados Unidos, Hall (2000 y 2001) compara el valor de las acciones, su deuda y el valor de su capital físico, y encuentra que formaron grandes cantidades de capital intangible en la segunda mitad del siglo $\mathrm{xx}$, ante todo en la última década. Argumenta que los intangibles aumentan el rendimiento de la inversión, la productividad y el valor de mercado de la firma. De modo que los intangibles se deberían considerar inversión porque aumentan el producto y tienen impacto de largo plazo.

\footnotetext{
${ }^{14}$ Como el capital físico es una mercancía homogénea que se transa en un mercado competitivo con información perfecta, el precio refleja su productividad.
} 


\section{CARACTERÍSTICAS DEL CAPITAL INTANGIBLE}

La literatura menciona varias características del capital intangible por las que merece un tratamiento diferente al del capital tangible. A continuación se comentan las más importantes ${ }^{15}$ :

a. En general, su producción no es masiva. Webster $(2002,11)$ señala que, en gran parte, el capital intangible se produce dentro de la firma, $y$, a diferencia del capital tangible que se fabrica en masa y se compra como cualquier mercancía, es heterogéneo y difícil de sustituir o replicar. Por ello, es una fuente sostenible de ventaja competitiva para la firma (Lorente, 2000). Caballero $(1998,849)$ sostiene que las acciones de otras firmas funcionan como mecanismo de información cuando la inversión es irreversible. Si el capital intangible es heterogéneo y no se produce en forma masiva, la firma no puede utilizar antecedentes y eso dificulta el cálculo de la rentabilidad. Además, en el caso del capital físico, el inversionista conoce al menos su valor intrínseco, lo que no ocurre con el capital intangible. Así, la inversión en intangibles está sujeta a mayor incertidumbre que la inversión en capital físico.

b. La firma no se puede apropiar todo el rendimiento de la inversión porque los bienes de capital intangible tienen características de bienes públicos y no siempre es claro quién tiene los derechos de propiedad (Lorente, 2000); pueden existir entonces problemas de incentivos para producirlo y adquirirlo. E1 cuadro 2 clasifica los bienes de capital intangible en términos de rivalidad y exclusión.

Por ejemplo, una vez se elabora una fórmula para un nuevo producto, el hecho de que una firma la use no impide que otra pueda usarla, de modo que es un bien no rival aunque existan mecanismos legales de exclusión, como una patente. Si no existen mecanismos de exclusión adecuados, los competidores pueden apropiarse de ella, aunque no hayan incurrido en ningún costo para desarrollarla. Por esta razón el capital de producción aparece tanto en el caso de bienes excluibles como en el de no excluibles.

Así mismo, los conocimientos que adquiere un empleado cuando se capacita producirán rendimientos a la firma mientras haga parte de ella. Las horas que trabaja allí las puede ofrecer a otra, pero la firma no puede impedir que la competencia lo contrate posteriormente o que

${ }_{15}$ No todos los activos intangibles poseen estas características, aunque la literatura no lo hace explícito. Por ejemplo, si se adquiere software no elaborado específicamente para la firma, debe tener un mercado, lo que implica que es un producto homogéneo, con un precio conocido, y que su valoración no es un problema. Además, no es un activo específico de la firma, porque cualquiera lo puede comprar. Otro ejemplo es de las patentes, donde no hay conflicto de propiedad. 
utilice esos conocimientos en otras actividades fuera del horario de trabajo. Así, el capital humano es no rival, pero puede no estar sujeto a exclusión. Y parte del rendimiento de la inversión en este capital no va a la firma que invirtió en él porque no tiene control total del elemento en que está almacenado ese capital, en este caso, el trabajador.

Cuadro 2

Tipos de bienes y de capital intangible

\begin{tabular}{lll}
\hline & Excluible* & No excluible \\
\hline \multirow{3}{*}{ Rival } & Capital organizacional & Capital humano \\
& Inversión óptima & Inversión insuficiente \\
& Uso óptimo & Sobre utilización \\
\multirow{2}{*}{ No rival } & Capital de producción & Capital de producción \\
& Inversión óptima & Capital relacional o de mercado \\
& Subutilización & Inversión insuficiente \\
& & Uso óptimo \\
\hline
\end{tabular}

* Debido a derechos de propiedad legales.

Fuente: Webster $(2006,86)$.

c. Es difícil conocer el valor del acervo de intangibles. Por definición no tienen materia y es difícil hacer un inventario claro de sus componentes, y, si se pudiese, no sería fácil determinar su valor porque, por su heterogeneidad y la falta de mercado, no hay precio.

d. Hay alta complementariedad entre intangibles y entre estos y el capital fijo. Ducharme (1998, 5), quien cita a Mincer (1989), lo muestra para el capital humano y la innovación. Según él, el capital humano es un factor productivo en sí mismo y contribuye al proceso de innovación en la firma (en términos de Webster, al capital de producción). Y Peneder (2002) encuentra relaciones de complementariedad entre capital humano y recursos tecnológicos.

e. Vosselman (1998, 10-11) señala que parte de los intangibles se adquiere con los bienes de capital fijo o está asociada directamente a ellos. Por ejemplo, cuando se compra una máquina, es común que se incluya un curso sobre cómo manejarla, o cuando se compra un computador, que tenga instalado algún software. Brynjolfsson et al. (2002) reconocen este hecho y encuentran relaciones de complementariedad entre adquisición de tecnologías de información (medida por la compra de computadores) y capital organizacional. Rodríguez y Araújo (2002) asocian las relaciones de complementariedad a la dificultad de identificar los diferentes tipos de intangibles de la firma, que lleva a valorarlos en conjunto. Señala además que, a diferencia del capital físico, los intangibles que no se usan, no se reconocen ni se valoran (el capital fijo al menos tiene valor por sus materiales). $\mathrm{Y}$ es posible que cierto intangible solo se reconozca y se use junto 
con otros intangibles (p. ej., conocimiento de sistemas cuando se usa software).

\section{INVERSIÓN EN INTANGIBLES Y ESTRATEGIA COMPETITIVA}

En las secciones anteriores se definió el capital intangible, se mostró su importancia actual y se mencionaron sus características. Pero no se esbozó ninguna hipótesis sobre por qué las firmas invierten en este tipo de capital. Aunque en la teoría no hay consenso sobre si es o no un factor productivo, en adelante se supone que sí lo es, y esa es una razón para que las firmas dediquen recursos a su adquisición. Cabe preguntar, sin embargo, cómo deciden entre varias alternativas de inversión y por qué eligen capital intangible.

Como ya se sugirió, la teoría responde que escogen la que genera mayor rendimiento esperado, y eligen intangibles porque su rentabilidad esperada es mayor. Según Porter la respuesta es que invierten para mantener una ventaja competitiva, de modo que para maximizar los beneficios tratan de limitar la competencia; el mecanismo para ello es la estrategia competitiva ${ }^{16}$. Se distinguen varias estrategias: de precios (valor agregado medio o bajo y precio bajo), de diferenciación (valor agregado alto y precio medio, para productos únicos con diseño e investigación), híbrida (bien diferenciado con precio menor al de la competencia), de segmentación en diferenciación (valor agregado $\mathrm{y}$ precios altos) y de fracaso (precio alto y bajo valor agregado, que solo funcionan en caso de monopolio). Según sea la estrategia, los intangibles permiten obtener costos bajos o diferenciación, y ambos limitan la competencia ${ }^{17}$.

Lo que es coherente con las características de los intangibles. Si el capital intangible es un bien heterogéneo que no se produce en masa, la firma que lo consiga puede realizar el proceso productivo, incluido todo lo que se requiere antes de fabricar el bien o prestar el servicio, hasta obtener beneficios, de un modo difícil de imitar para sus competidores. Sin embargo, si el capital intangible tiene características de bien público, la firma debe asegurar mecanismos de exclusión adecuados para apropiarse los beneficios. El mercadeo, la publicidad y las actividades que mejoran los aspectos organizacionales se pueden considerar como inversión cuando se considera el proceso productivo en este sentido amplio, porque reportan beneficios (como muestra la evidencia empírica), no solo aumentando la disposición a pagar,

${ }^{16}$ Porter la define así: "emprender acciones ofensivas o defensivas para crear una posición defendible en un sector, enfrentar con éxito las cinco fuerzas competitivas y obtener así un rendimiento mayor sobre la inversión" $(1986,55)$.

${ }^{17}$ Johnson y Scholes (2001) describen las características de cada estrategia. 
lo que indica que la mercancía es más valiosa, sino alimentando los recursos reputacionales que reducen costos de transacción ${ }^{18}$.

Si para maximizar sus beneficios la firma puede limitar la competencia, la inversión es clave. Dixit (1980) muestra que puede crear poder de monopolio con capacidad instalada, porque ante posibles competidores podrá reducir el precio hasta un nivel imposible para ellos. Así, la inversión puede ser parte de la estrategia competitiva. En el caso de los intangibles, como señalan Lorente (2000), Webster (2002), David (2004) ${ }^{19}$ y Slywotzky et al. (2004), el poder de monopolio de una firma obedece a que sus competidores no pueden conseguir tal capital intangible porque no se compra ni se vende en el mercado. Además, en modelos de crecimiento endógeno (como el de Romer, 1990), algunas características de bienes públicos como la no rivalidad pueden generar rendimientos crecientes y, por tanto, situaciones de monopolio.

El modelo que se presenta a continuación supone que las firmas deben fabricar capital intangible si quieren usar más del inicial, el cual es el mismo de sus rivales. Aunque no considera la incertidumbre y capta la heterogeneidad mediante costos de producción del capital intangible distintos y no como diferencias en la fabricación del bien final, los beneficios de dos firmas diferirán aunque el costo marginal del bien final sea igual.

\section{GENERALIDADES}

En esta sección se presenta un modelo de competencia à la Cournot que muestra los incentivos de la firma para invertir en capital intangible. Este es un factor fijo que puede ser utilizado libremente por todas las firmas, y si alguna requiere una cantidad adicional, la puede fabricar para su uso exclusivo (sin comercializarla). La firma pertenece a una industria con $n$ firmas que compiten con productos homogéneos. Sigh y Vives (1984) demuestran que competir à la Cournot es una estrategia dominante frente a competir à la Bertrand. Como los productos son homogéneos, la única estrategia disponible es buscar un precio bajo. La firma enfrenta una función de producción:

${ }^{18}$ Slywotzky et al. $(2004,55)$ dan el siguiente ejemplo: "supongamos que quiere construir un nuevo negocio de información, vender servicios para ayudar a las compañías a manejar sus aplicaciones de software. El comprador directo es el director de informática de cualquier compañía grande. Si llama por teléfono a 100 directores de informática, ¿cuántos le devolverán la llamada el mismo día? Si es de гвм, la respuesta es 100 . Si es una compañía novata, la respuesta es ninguno. La diferencia se expresa en meses de demora en la consecución de clientes, en ingresos perdidos o pospuestos y en una reducción de las utilidades".

${ }_{19}$ David se refiere en particular al caso de la I\&D. 
$q_{i}=k^{\alpha} l^{1-\alpha} I_{i}$

donde $k$ es el capital físico, $l$ el trabajo e $I_{i}$ el capital intangible ${ }^{20}$. Si $I_{i}$ es fijo, la función de producción muestra rendimientos constantes a escala, y el costo marginal de la firma $i, c_{i}$, es constante, pero si $I_{i}$ se puede elegir, la función de producción tendrá rendimientos crecientes a escala. Por ahora se supone que $I_{i}$ es constante. Así, las $n$ firmas enfrentan una función de demanda lineal inversa:

$p=a-b Q$

donde $Q$ es la cantidad total de producto $\left(Q=\sum_{i=1}^{n} q_{i}\right), b$ mide el impacto sobre el precio de un cambio en la cantidad demandada por cualquier firma $^{21}$. E1 problema de la firma $i$ es:

$\max \prod_{q_{i}}=\left(a-b q_{i}-b \sum_{j \approx i} q_{j}\right) q_{i}-c_{i} q_{i}$

cuya solución se presenta en el Anexo 1. Las cantidades que producen i y una firma representativa de las $n-1$ restantes están dadas por:

$q_{i}^{*}=\frac{a-n c_{i}+(n-1) \bar{c}_{j}}{b(n+1)}$

$\bar{q}_{i}^{*}=\frac{a-2 \bar{c}_{j}+c_{i}}{b(n+1)}$

donde $\bar{q}_{j}$ y $\bar{c}_{j}$ representan el producto y el costo marginal promedio de esas $n-1$ firmas. La cantidad de bien final que produce la firma $i$ depende negativamente de su costo marginal y positivamente del costo de la otra firma, pero el efecto de cambios en dichas variables no es simétrico. Un incremento unitario de su costo marginal reduce la cantidad producida en $n / b(n+1)$, pero un aumento de igual magnitud en el costo marginal promedio aumenta el producto en $(n-1) / b(n+1)$; es decir, el impacto de una reducción en el costo propio es proporcionalmente mayor que un aumento en el costo de las rivales. Así, es difícil que el aumento de producción ocasionado por una reducción del costo marginal propio sea anulado por las firmas rivales. Para que eso ocurra en un duopolio, por ejemplo, la firma rival debe reducir el doble de su costo marginal. Al comparar la producción de la firma $i$ y de la firma representativa, se ve que si $i$ tiene un costo marginal inferior al promedio de la industria, la cantidad producida es mayor que el promedio. El precio de equilibrio

20 El exponente de $I_{i}$ es 1 por simplicidad, pero los resultados no cambian significativamente cuando el exponente es diferente de 1. La función de costos cuando $I_{i}$ permanece fijo podría ser homogénea de grado menor o mayor que 1 , pero por simplicidad se supone homogénea de grado 1.

${ }^{21}$ El coeficiente $b$ es igual para todas porque los productos son homogéneos. 
$p^{*}=\frac{a+c_{i}+(n-1) \bar{c}_{j}}{(n+1)}$

es afectado de igual forma si cambia el costo marginal de cualquiera de las firmas. El margen precio-costo es mayor que el promedio para la firma con menor costo marginal. Los beneficios, para la firma $i \mathrm{y}$ para la firma promedio, son:

$\Pi_{i}^{*}=\frac{1}{b}\left[\frac{a-n c_{i}+(n-1) \bar{c}_{j}}{(n+1)}\right]^{2}$

$\bar{\Pi}^{*}=\frac{1}{b}\left[\frac{a-2 \bar{c}_{j}+c_{i}}{(n+1)}\right]^{2}$

Es decir, dependen negativamente del costo marginal propio. Eso significa que, si el costo marginal de $i$ es mayor que el promedio, sus beneficios son menores; y viceversa. Además, así como ocurre con la cantidad, las variaciones del costo marginal tienen efectos asimétricos en los beneficios. Lo que se gana al lograr mayor participación de mercado compensa lo que se pierde por vender a menor precio.

\section{EL CAPITAL INTANGIBLE EN EL MODELO DE COURNOT}

Hasta el momento se ha mostrado que cuando las firmas compiten à la Cournot con bienes homogéneos, obtienen una ventaja competitiva aquellas que consiguen un costo marginal inferior al del promedio. La función de producción $q_{i}=k^{\alpha} l^{1-\alpha} I_{i}$ es una función de producción en sentido amplio, es decir, incluye lo que la firma necesita desde la organización del proceso productivo hasta la venta del producto, no solo lo que requiere para producir los bienes finales. La firma recibe ingresos cuando los vende y solo entonces puede obtener beneficios. Si lo que ocurre antes o después de la producción es costoso, la posibilidad de alcanzar beneficios se reduce. Como lo plantea Coase, la firma es más que un ente que produce bienes, es un sistema que organiza transacciones por fuera del mercado. Estas también consumen recursos y hacen posible que la producción de bienes reporte beneficios a la firma. Así, la firma solo puede crecer hasta el punto en que el costo de organizar una transacción extra dentro de la firma es igual al de llevarla al mercado (Coase, 1937, 394). Si $I_{i}$ es fijo, su función de costos tiene la forma:

$C T_{i}=d q_{i} / I_{i}$

donde $d$ es una constante que depende del salario, la tasa de interés y las ponderaciones del capital y el trabajo, los cuales son iguales 
para todas las firmas ${ }^{22}$. Como el costo marginal es $c^{i}=d / I$, una mayor cantidad de intangibles lo reduce, lo cual es coherente con la función de producción en sentido amplio. Algunos aumentos en el capital intangible-mejoras en los procesos y de comunicación o innovaciones menores, p. ej.- pueden reducir los costos de las operaciones al interior de la firma, que no son costos de producción sino de transacción. Si $I_{i}$ aumenta, el costo marginal cae y el producto crece.

Siguiendo a Webster (2002), ningún proceso productivo puede llevarse a cabo sin capital intangible. Supongamos que al inicio todas las firmas comparten un nivel de capital intangible $\bar{I}$, el cual usan libremente. $\bar{I}$ es un bien público que incluye conocimiento común (p. ej., una receta genérica), entrenamiento promedio de la mano de obra, instituciones públicas, leyes, etc. En cierto momento y por una sola vez, la firma puede decidir invertir cierta cantidad de recursos $k_{i}^{I}$, que en este caso son capital físico, para producir capital intangible y emplearlo en la fabricación de bienes finales. Como ya se mencionó, el capital intangible se fabrica internamente y no se comercializa. Así, si la firma desea un nivel mayor que $\bar{I}$ debe elaborarlo. En este caso suponemos una función de producción lineal:

$I=\eta_{i} k_{i}^{I}$

donde $\eta_{i} \in[0, \infty)$ capta la productividad de la firma $i$ en la fabricación de capital intangible. Este parámetro representa qué parte de los recursos que invierte la firma en capital intangible se convierte en factor de producción y cuánto simplemente se gasta ${ }^{23}$. Se toma el intervalo $[0, \infty)$ porque no se descarta la posibilidad de que cada unidad de capital físico se convierta en más de una unidad de capital intangible, pero sí se supone que no se puede convertir en una suma negativa. Si $\eta_{i}$ es diferente para todas las firmas, el costo de producción del capital intangible también es distinto. Así, si la firma decide invertir en capital intangible considerando que dispone del nivel común $\bar{I}$, al final tendrá $\bar{I}+\eta_{i} k_{i}^{I}$, y el costo marginal de producir bienes finales será:

$c_{i}=\frac{d}{\left(\bar{I}+n_{i} k_{i}\right)}$

${ }^{22}$ Varian (1992, 79-83) muestra el desarrollo para el caso de dos firmas.

${ }^{23}$ Aunque otra característica de la inversión en capital intangible mencionada en la literatura es la incertidumbre, que se puede interpretar en función de $\eta$ suponiendo que es una variable aleatoria, por simplicidad se toma aquí como un parámetro conocido por la firma (no necesariamente por las rivales). 
Por simplicidad, suponemos que la vida útil del capital intangible es de $N$ periodos, sin importar el tipo de bien ni la firma ${ }^{24}$. No se hace ningún supuesto sobre la evolución del acervo de intangibles en ese lapso, por ejemplo, no se especifica si la depreciación es gradual o no, porque el análisis es estático, y el momento que se analiza es aquel donde la cantidad de intangibles es $\bar{I}+n_{i} k_{i}^{I}$. Como la inversión para producir capital intangible es por una sola vez y este luego se convierte en un factor fijo, los recursos invertidos son un costo irrecuperable. Pero los ingresos de la firma deben cubrir dicho costo. Suponemos que la firma cubre el monto invertido $k_{i}^{I}$ pagando una cuota fija en cada uno de $\operatorname{los} N$ periodos en los que ese factor es productivo ${ }^{25}$, dada por:

$k_{i}^{I} \frac{(1+r)^{N} r}{(1+r)^{N}-1}$

Con la inversión en $I$ la firma reduce su costo marginal, pero aumenta el costo medio por ese costo fijo. La situación dentro de la firma se asemeja a la de un monopsonio porque al departamento productor de $I$ solo se le reconocen los costos en que incurrió para elaborar tal factor productivo ${ }^{26}$. Si la firma que debe decidir si invierte o no en capital intangible conoce el valor de $\eta_{i}$, el de sus rivales y el monto que dedican a producir intangibles (o lo conjetura), sabe qué beneficios obtendrá para cada valor posible de $k_{i}^{I}$, dados por la expresión:

$$
\Pi_{i}=\frac{1}{b(n+1)^{2}}\left[a-n \frac{d}{\bar{I}+\eta_{i} k_{i}^{I}}+(n+1) \frac{d}{\bar{I}+\bar{\eta} k_{i}^{I}}\right]^{2}-K_{i}^{I} \frac{(1+r)^{N} r}{(1+r)^{N}-1}
$$

donde $\bar{\eta} \bar{k}_{j}^{I}$ es la conjetura de $i$ sobre la productividad media de la industria en la producción de capital intangible multiplicada por el monto promedio dedicado a ese fin. Cuando $i$ no invierte en capital intangible $\bar{k}_{i}^{I}=0$. El cuadro 3 muestra la cantidad óptima, el precio

\footnotetext{
${ }^{24}$ Esto supone que al cabo de los $N$ periodos el capital intangible se ha depreciado totalmente o pasa a formar parte del nivel común. Aunque autores como Slywotzky et al. (2004) afirman que los intangibles aumentan a medida que se utilizan, es decir, que se aprecian y no se deprecian.

${ }_{25}$ En el Anexo 2 se desarrolla esta expresión.

${ }^{26}$ En realidad, la situación es de monopolio bilateral: el departamento que produce capital intangible es el único oferente para el que produce bienes finales, y este es el único comprador. Pero si, como se supone aquí, la firma solo reconoce los costos en que incurre para obtener capital intangible, la situación es de monopsonio y los beneficios adicionales que este genere los captará en su totalidad el departamento que produce bienes finales. Puede ocurrir que al departamento que produce bienes intangibles se le reconozcan beneficios de monopolio, como en Romer (1990).
} 
de mercado y los beneficios de la firma i, en monopolio, duopolio, oligopolio y competencia perfecta ${ }^{27}$.

Como se puede observar, aun en competencia perfecta los beneficios pueden ser mayores que 0 . Para la firma $i$, la cantidad producida depende de la diferencia entre el costo marginal promedio de la industria y el suyo. Si $c_{i}=\bar{c}$ se obtiene el resultado tradicional del modelo de Cournot en el que $q_{i}$ tiende a 0 . En cuanto al precio, como los bienes son homogéneos, la firma $i$ toma el precio de mercado, $\bar{c}$,y si $c_{i}<\bar{c}$ la firma $i$ tendrá un margen precio-costo positivo y, por tanto, beneficios antes de cubrir los costos fijos.

Cuadro 3

Cantidad óptima, precio y beneficios con inversión en capital intangible

\begin{tabular}{|c|c|c|c|}
\hline & Cantidad & Precio & Beneficios \\
\hline 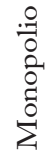 & $\frac{1}{b}\left[a-\frac{d}{\bar{I}+\eta_{i} k_{i}^{I}}\right]$ & $\frac{1}{2}\left[a+\frac{d}{\bar{I}+\eta_{i} k_{i}^{I}}\right]$ & $\frac{1}{4 b}\left[a-\frac{d}{\bar{I}+\eta_{i} k_{i}^{I}}\right]^{2}-k_{i}^{I} \frac{(1+r)^{N} r}{(1+r)^{N}-1}$ \\
\hline \begin{tabular}{l}
$:$ \\
\hdashline \\
0 \\
0 \\
0 \\
0
\end{tabular} & $\frac{1}{b}\left[a-\frac{2 d}{\bar{I}+\eta_{i} k_{i}^{I}}+\frac{d}{\bar{I}+\overline{\eta_{j} k_{j}^{I}}}\right]$ & $\frac{1}{3}\left[a+\frac{d}{\bar{I}+\eta_{i} k_{i}^{I}}+\frac{d}{\bar{I}+\overline{\eta_{j} k_{j}^{I}}}\right]$ & $\begin{array}{l}\frac{1}{9 b}\left[a-\frac{2 d}{\bar{I}+\eta_{k_{i}^{I}}^{I}}+\frac{d}{\bar{I}+\overline{\eta_{j} \bar{k}_{j}^{I}}}\right] \\
-k_{i}^{I} \frac{(1+r)^{N} r}{(1+r)^{N}-1}\end{array}$ \\
\hline $\begin{array}{l}: \\
0 \\
0 \\
01 \\
: 0 \\
07\end{array}$ & $\frac{1}{b(n+1)}\left[a-\frac{n d}{\bar{I}+\eta_{i} k_{i}^{I}}+\frac{(n-1) d}{\bar{I}+\overline{\eta_{j}} \bar{k}_{j}^{I}}\right]$ & $\frac{1}{n+1}\left[a+\frac{d}{\bar{I}+\eta_{i} k_{i}^{l}}+\frac{(n-1) d}{\bar{I}+\overline{\eta_{j}} \overline{k_{j}^{I}}}\right]$ & $\begin{array}{l}\frac{1}{b(n+1)^{2}} \\
{\left[a-\frac{n d}{\bar{I}+\eta_{i} k_{i}^{I}}+\frac{(n-1) d}{\bar{I}+\overline{\eta_{j}} \overline{k_{j}^{I}}}\right]^{2}} \\
-k_{i}^{I} \frac{(1+r)^{N} r}{(1+r)^{N}-1}\end{array}$ \\
\hline 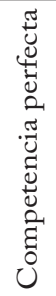 & $\frac{1}{b}\left[\frac{d}{\bar{I}+\overline{\eta_{j} k_{j}^{I}}}-\frac{d}{\bar{I}+\eta_{i} k_{i}^{I}}\right]$ & $\frac{d}{\bar{I}+\overline{\eta_{j} \bar{k}_{j}^{I}}}$ & $\begin{array}{l}\frac{d^{2}\left(\overline{\eta_{j} k_{j}^{I}}-\eta_{i} k_{i}^{I}\right)^{2}}{b\left(\bar{I}+\overline{\eta_{j}} \overline{k_{j}^{I}}\right)^{2}\left(\bar{I}+n_{i} k_{i}^{I}\right)^{2}} \\
-k_{i}^{I} \frac{(1+r)^{N} r}{(1+r)^{N}-1}\end{array}$ \\
\hline
\end{tabular}

\section{¿CUÁNTO SE DEBE INVERTIR EN CAPITAL INTANGIBLE? ANÁLISIS GRÁFICO}

E1 problema de la firma es elegir $K_{i}^{l}$ para maximizar sus beneficios:

${ }^{27}$ Para el caso de monopolio se tomó $n=1$, para duopolio $n=2$ y para competencia perfecta se calculó el límite cuando $n \rightarrow \infty$. 
$\max K_{i}^{I} \frac{1}{b(n+1)^{2}}\left[a-n \frac{d}{\bar{I}+\eta_{i} k_{i}^{I}}+(n-1) \frac{d}{\bar{I}+\bar{\eta} \overline{k_{j}^{I}}}\right]^{2}-K_{i}^{I} \frac{(1+r)^{N} r}{(1+r)^{N}-1}$

El problema se aborda gráficamente porque la solución analítica es extensa y de difícil interpretación. La función de beneficios de la firma se puede descomponer en tres:

$$
\begin{aligned}
& \frac{1}{b(n+1)^{2}}\left[a-n \frac{d}{\bar{I}+\eta_{i} k_{i}^{I}}+(n-1) \frac{d}{\bar{I}+\bar{\eta} \overline{k_{j}^{I}}}\right] \\
& {\left[a-n \frac{d}{\bar{I}+\eta_{i} k_{i}^{I}}+(n-1) \frac{d}{\bar{I}+\bar{\eta} \overline{k_{j}^{I}}}\right]} \\
& -k_{i}^{I} \frac{(1+r)^{N} r}{(1+r)^{N}-1}
\end{aligned}
$$

La gráfica 1 muestra la ecuación 13 suponiendo que $K_{i}^{I}$ puede tomar cualquier valor real. La ecuación 14 tiene una forma similar a la 13 pero no se divide por $b(n+1)^{2}$. El producto de las dos funciones, los beneficios de la firma antes de cubrir los costos fijos, es:

$$
\frac{1}{b(n+1)^{2}}\left[a-n \frac{d}{\bar{I}+\eta_{i} k_{i}^{I}}+(n-1) \frac{d}{\bar{I}+\bar{\eta} \overline{k_{j}^{I}}}\right]^{2}
$$

Gráfica 1

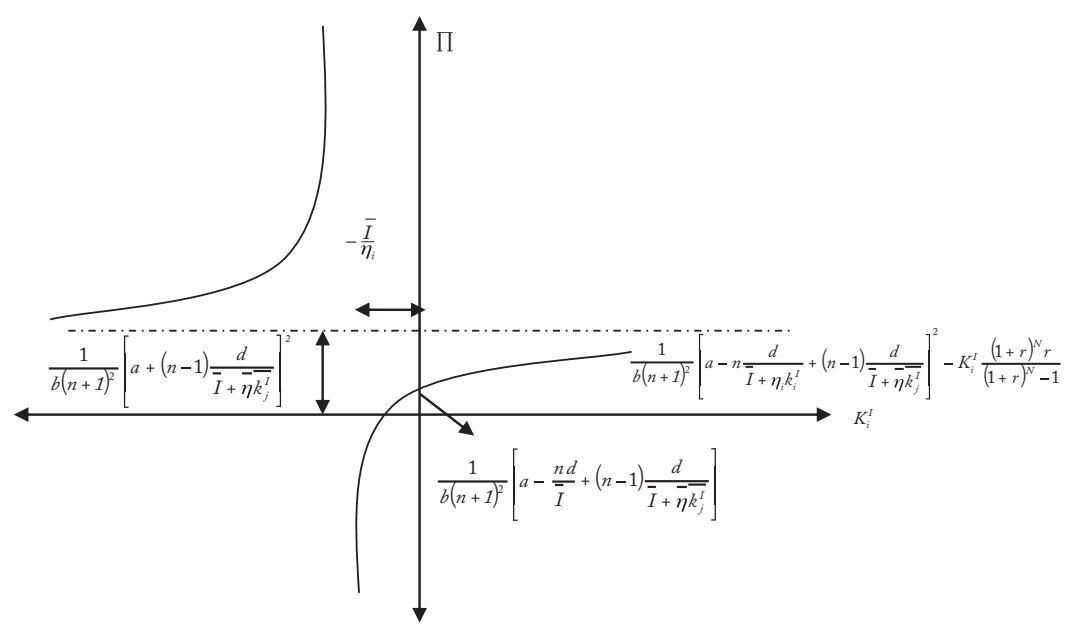




\section{Gráfica 2}

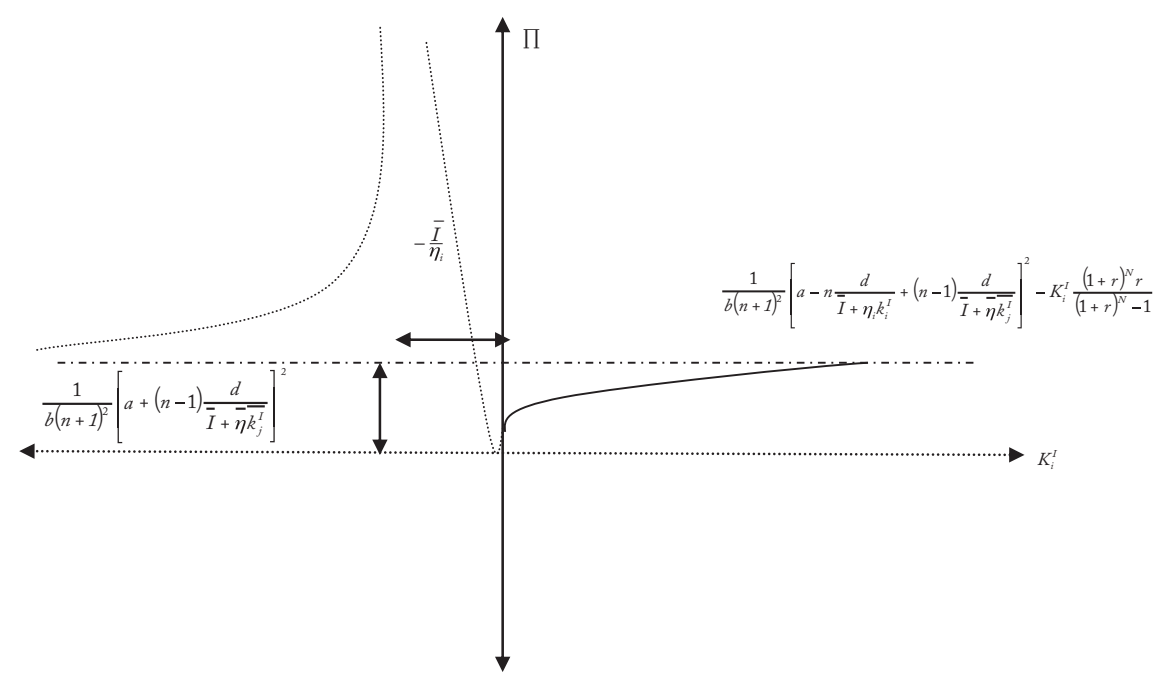

Gráfica 3

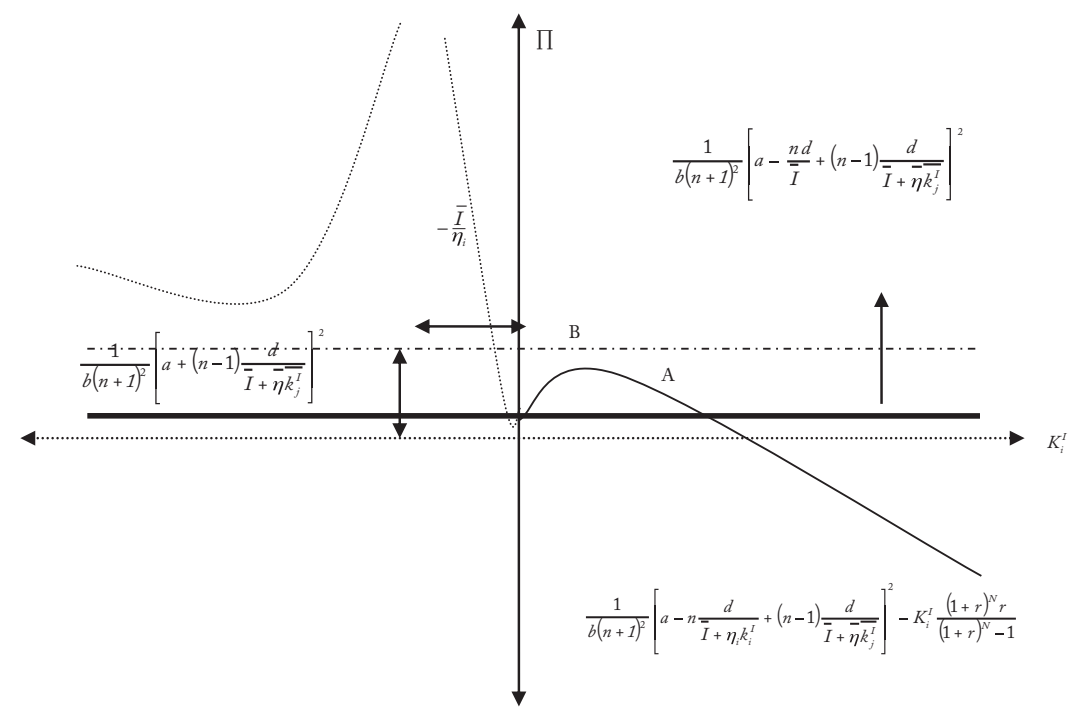

La gráfica 2 muestra la ecuación 16. La línea punteada representa los valores de la función de beneficios cuando $K_{i}^{I}<0$, que no tienen interpretación en este problema ${ }^{28}$. La ecuación 15 es una recta con pendiente negativa menor que 1 y pasa por el origen; así, al sumar las

${ }^{28}$ Porque el capital intangible no se transa en un mercado, y una vez producido, la única manera de reducir su acervo es que se deprecie o pase a ser parte del capital intangible común. 
ecuaciones 15 y 16, el punto de corte con el eje vertical no cambia. La suma de 15 y 16, la función de beneficios, se representa en la gráfica 3. La línea gruesa muestra los beneficios de $i$ si decide no invertir en $I$; el punto $A$ muestra hasta qué valor de $K_{i}^{I}$ es rentable invertir en capital intangible y el punto $B$ marca el máximo de la función de beneficios de $i$, dada su conjetura sobre el capital intangible del resto de la industria.

\section{¿DE QUÉ DEPENDE LA PENDIENTE DE LA FUNCIÓN DE BENEFICIOS?}

Los valores de $K_{i}^{I}$ para los que es rentable que la firma $i$ invierta en capital intangible forman un conjunto cerrado, $(O, A)$. Este conjunto no es vacío siempre y cuando existan valores de $K_{i}^{I}$ para los cuales la pendiente de la ecuación 16, los beneficios de la firma después de pagar costos variables y antes de pagar los fijos, sea mayor que la constante $\frac{(1+r)^{v} r}{(1+r)^{v}-1}$. El valor de $K_{i}^{I}$ que maximiza los beneficios es aquel donde las dos pendientes se igualan. Cuanto mayor es la pendiente de la ecuación 16, mayores son los beneficios que obtiene la firma cuando invierte, más amplio el rango en el que es rentable invertir y mayor el $K_{i}^{I}$ óptimo. Por ello es necesario analizar de qué depende esa pendiente.

El principal determinante de la pendiente es $\eta_{i}$, el único parámetro que no comparten las firmas. Cuando $\eta_{i} \rightarrow 0$, la pendiente también es 0 . Eso significa que si la productividad de $i$ en la fabricación de capital intangible es muy baja, la firma nunca tendrá incentivos para dedicar recursos a este tipo de inversión. La pendiente depende negativamente del capital intangible promedio de la industria. De modo que los incentivos de la firma $i$ para invertir en él disminuyen cuando sus competidores tienen mayor capital intangible.

En cuanto al número de firmas, el límite de la pendiente cuando $J \rightarrow 0$ es:

$$
\frac{2 d^{2} \eta_{i}\left(\eta_{i} k_{i}^{I}-\overline{n_{j}} \overline{k_{j}^{I}}\right)}{b\left(\bar{I}+\overline{\eta_{j}} \overline{K_{j}^{I}}\right)\left(\bar{I}+\eta_{i} k_{i}^{I}\right)^{3}}
$$

una expresión positiva siempre y cuando el capital intangible de la firma sea superior al promedio.

\section{¿CUÁNTO INVERTIR?}

La gráfica 3 indica que la firma tiene incentivos para invertir en capital intangible incluso una cantidad mayor a la óptima, porque hay un 
intervalo donde $K_{i}^{I}$ supera al óptimo y los beneficios son mayores que los que obtendría si $K_{i}^{I}=0$. Este resultado difiere de lo que plantea la literatura sobre bienes públicos o que generan externalidades, aunque en este caso valores superiores al óptimo no sean de equilibrio.

Para conocer el intervalo donde es rentable invertir en capital intangible se igualan los beneficios que la firma recibe por invertir un monto $K_{i}^{I}$ y por no invertir.

$$
\frac{1}{b(n+1)^{2}}\left[a-n \frac{d}{\bar{I}+\eta_{i} k_{i}^{I}}+(n-1) \frac{d}{\bar{I}+\bar{\eta} \overline{k_{j}^{I}}}\right]^{2}-k_{i}^{I} \frac{(i+r)^{N} r}{(i+r)^{N}-1}=\frac{1}{b(n+1)^{2}}\left[a-\frac{n d}{\bar{I}}+(n-1) \frac{d}{\bar{I}+\bar{\eta} \overline{k_{j}^{I}}}\right]^{2}
$$

Si $R=\frac{(i+r)^{N} r}{(i+r)^{N}-1}$

$\frac{1}{b(n+1)^{2}}\left[a-n \frac{d}{\bar{I}+\eta_{i} k_{i}^{I}}+(n-1) \frac{d}{\bar{I}+\bar{\eta} \overline{k_{j}^{I}}}\right]^{2}-k_{i}^{I} R=\frac{1}{b(n+1)^{2}}\left[a-\frac{n d}{\bar{I}}+(n-1) \frac{d}{\bar{I}+\bar{\eta} \overline{k_{j}^{I}}}\right]^{2}$

Se soluciona para $K_{i}^{I}$ y, como lo muestra la gráfica, se obtienen tres puntos donde los beneficios cuando invierte en intangibles son iguales a los que obtendría si no invierte, dado el promedio de la industria ${ }^{29}$.

De estos tres puntos, uno es $0 \mathrm{y}$, por definición, los beneficios deben ser iguales si la firma no invierte en intangibles o invierte una suma igual a 0 ; otro es negativo $y$, por tanto, se excluye; $y$ el otro pertenece al intervalo $[0, \infty)$, el cual determina la máxima suma que la firma estaría dispuesta a invertir en capital intangible (punto A, gráfica 3). Otra posibilidad es que no sea rentable invertir en intangibles en ninguna circunstancia. Como una de las soluciones es $K_{i}^{I}=0$, se puede inferir que cuando se igualan los pagos por invertir y por no invertir, en algún momento se tiene una ecuación de la forma $k_{i}^{t}\left[f\left(k_{i}^{t}\right)\right]$, donde $f\left(k_{i}^{t}\right)$ es una de grado 2. Si las raíces son números complejos, siempre será más rentable no invertir en capital intangible.

Esto ocurre cuando la pendiente de la ecuación 16 en el intervalo $[0, \infty)$ siempre es menor que $R$, es decir, cuando la función de beneficios de la firma si invierte en intangibles siempre está por debajo de la línea gruesa.

\footnotetext{
${ }^{29}$ Tampoco se presentan los resultados, porque sus expresiones son extensas y de difícil interpretación.
} 


\section{¿CUÁNTO INVERTIR PARA TENER LOS BENEFICIOS DE LA FIRMA PROMEDIO?}

Según el Anexo 1, los beneficios de la firma promedio están dados por: $\bar{\Pi}=\frac{1}{b(n+1)^{2}}\left[a+\frac{d}{\bar{I}+\eta_{i} k_{i}^{I}}-\frac{2 d}{\bar{I}+\bar{\eta} \overline{k_{j}^{I}}}\right]^{2}-\frac{\overline{k_{j}^{I}}(1+r)^{N} r}{(1+r)^{N}-1}$

En esta función, la asíntota vertical es la misma que la de la función de beneficios de la firma $i$, pero la horizontal es más baja que la ecuación 16, y para valores grandes de $\overline{k_{j}^{I}}$ podría ser negativa. Así, es posible que exista un intervalo de valores donde los beneficios de $i$ sean mayores que los de sus competidores. La gráfica 4 representa la ecuación 18 en el plano junto con los beneficios de la firma $i^{30}$.

Gráfica 4

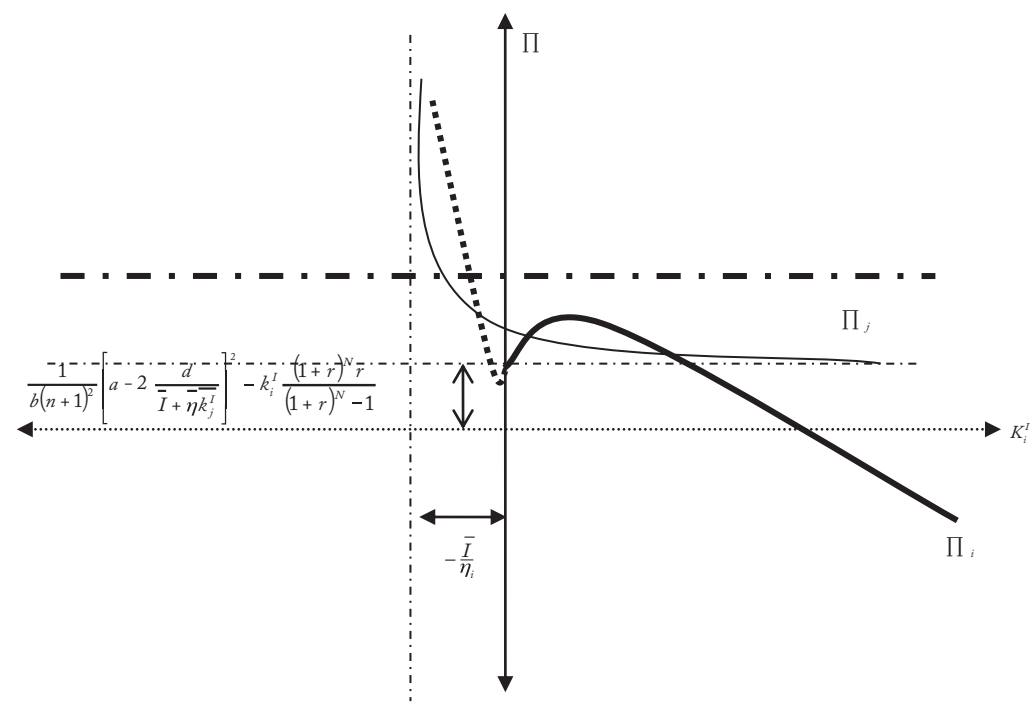

Las soluciones analíticas de este problema también son extensas, pero se pueden obtener gráficamente algunos resultados. La firma $i$ puede tener beneficios mayores o menores que sus rivales, según cuánto invierta en $I$. Si $\overline{k_{j}^{I}} \neq 0$ los beneficios de las dos firmas son distintos para $k_{i}^{I}=0^{31}$; además, diferencias de productividad en la fabricación de capital intangible podrían generar beneficios diferentes para las

${ }^{30}$ La gráfica solo presenta la función a la derecha de la asíntota vertical, porque ninguna firma puede invertir una suma negativa en capital intangible.

${ }^{31}$ Por ello, en la gráfica, las funciones de beneficios de las firmas $i$ y $j$ no se cruzan en el eje vertical sino algo más a la derecha. Si $\overline{k_{j}^{l}}=0$, dado que al inicio las firmas solo tienen el nivel común $\bar{I}$, cuando $k_{i}^{l}=0$ sus beneficios son iguales. 
firmas, aunque $k_{i}^{I}=\overline{k_{j}^{l}}$. También es posible que dado el valor de $\overline{k_{j}^{l}}$, no haya valores de $k_{i}^{l}$ que permitan igualar los beneficios de su rival.

Esto constata que el aumento del acervo de capital intangible hace posible que una firma obtenga beneficios mayores que los de sus rivales $y$, como plantea Dixit, que esa inversión impide la competencia perfecta. Si el acervo de intangibles de $i$ es igual al de la firma promedio, la diferencia de beneficios dependerá de cuánto invirtió cada una para alcanzar ese nivel. Así que lo más importante es la productividad de la firma en la fabricación del capital intangible.

\section{CONCLUSIONES}

E1 modelo de competencia à la Cournot con bienes homogéneos que presenta este artículo incluye el capital intangible como un factor fijo necesario en el proceso productivo, y todas las actividades anteriores y posteriores a la fabricación del bien final que llevan a obtener beneficios. Hay una cantidad común de capital intangible que puede ser utilizada libremente por todas las firmas de una industria; si una firma requiere una cantidad adicional, la debe fabricar para uso exclusivo. Como en este modelo es un factor fijo, el capital intangible específico de la firma reduce su costo marginal, pero aumenta los costos fijos. La firma debe encontrar entonces el nivel óptimo de inversión teniendo en cuenta esa disyuntiva.

El modelo muestra que, si la firma es suficientemente productiva en la fabricación de capital intangible, existe un nivel óptimo de inversión en intangibles mayor que 0 , el cual maximiza sus beneficios. Y que la firma tiene incentivos para invertir sumas superiores a la óptima, aunque no sean valores de equilibrio. Esto difiere de lo que se suele afirmar acerca de bienes con características de bien público, y se deriva de suponer que existen mecanismos eficientes de exclusión. Aunque existan muchas firmas, una firma puede obtener beneficios mayores que sus rivales siempre y cuando logre un costo marginal menor sin incurrir en costos fijos muy altos. En suma, la firma puede obtener una ventaja competitiva que se traduce en poder de mercado, bien se mida como participación en la oferta total o como margen precio-costo.

La rentabilidad de la inversión en intangibles depende básicamente de la productividad de la firma en su fabricación. En el modelo ese parámetro es exógeno, y queda para futura investigación saber de qué depende. Unas posibles extensiones serían incorporar la incertidumbre acerca del rendimiento y el nivel de capital intangible e incluir la diferenciación como estrategia competitiva. 


\section{ANEXO 1}

\section{COURNOT CON $n$ FIRMAS}

La firma pertenece a una industria con $j$ firmas cuyo producto es homogéneo y compiten à la Cournot. Si enfrentan una función de demanda lineal:

$p=a-b Q$

donde $Q$ es el producto total, $\Sigma q_{i}$, el problema de maximización de los beneficios de la firma $i$ es:

$\max _{q_{i}} \pi=\left(a-b q_{i}-b \sum_{j \neq i}^{n} q_{i}\right) q_{i}-c_{i} q_{i}$

cuya condición de primer orden es:

$a-2 b q_{i}-b \sum_{j \neq i} q_{j}-c_{i}=0$

$\frac{a-c_{i}}{2 b}-\frac{\sum_{j=i} q_{j}}{2}=q_{i}^{;}$

De modo análogo, para una firma $m$ representativa de la industria, con un costo marginal igual al promedio de las firmas diferentes de $i$, la condición de primer orden se puede escribir así:

$a-2 b q_{m}-b \sum_{j \times m} q_{j}-c_{j}=0$

$a-b q_{m}-b q_{i}-b \sum_{j \neq i} q_{j}-c_{j}=0$

Despejando $q_{i}$ e igualando esas dos condiciones se obtiene:

$$
\frac{a-b \sum_{j \neq i} q_{j}-c_{i}}{2 b}=\frac{a-b q_{m}-b \sum_{j \neq i} q_{j}-c_{j}}{b}
$$

Como la firma $m$ es representativa de la industria (excluyendo a la firma $i$ ), su costo marginal es el promedio de las $j-1$ restantes y $q_{m}$ se puede remplazar $\operatorname{por}_{q}^{-}$(el producto promedio) y la suma del producto de esas firmas por $(n-1) q_{j}$.

$$
\begin{aligned}
& \frac{a-(n-1) b q_{j}-c_{i}}{2 b}=\frac{a-b \bar{q}-(n-1) b \bar{q}-\bar{c}}{b} \\
& \frac{a-(n-1) b \bar{q}-c_{i}}{2 b}=\frac{a-n b \bar{q}-\bar{c}}{b}
\end{aligned}
$$




$$
\begin{aligned}
& a-(n-1) b \bar{q}-c_{i}=2 a-2 n b \bar{q}-2 \bar{c} \\
& \overline{b q}(2 n-n+1)=a-2 \bar{c}+c_{i} \\
& \bar{q}=\frac{a-2 \bar{c}+c_{i}}{b(n+1)}
\end{aligned}
$$

\section{Reemplazando en $q_{i}$ :}

$$
\begin{aligned}
& q_{i}=\frac{a-c_{i}}{2 b}-\frac{(n-1)}{2}\left[\frac{a-2 \bar{c}+c_{i}}{b(n+1)}\right] \\
& q_{i}=\frac{a(n+1)-c_{i}(n+1)-a(n-1)+2 \bar{c}(n-1)-c_{i}(n-1)}{2 b(n+1)} \\
& q_{i}=\frac{2 a-2 n c_{i}+2(n-1) \bar{c}}{2 b(n+1)} \\
& q_{i}=\frac{a-n c_{i}+(n-1) \bar{c}}{b(n+1)}
\end{aligned}
$$

Para el precio de equilibrio, $q_{i}$ y $\bar{q}$ se remplazan en la función de demanda:

$$
\begin{aligned}
& p=a-b\left[\frac{a-n c_{i}+(n-1) \bar{c}}{b(n+1)}\right]-b(J-1)\left[\frac{a-2 \bar{c}+c_{i}}{b(n+1)}\right] \\
& p=\frac{(n+1) a-a+n c_{i}-(n-1) \bar{c}-(n-1) a+(n-1) 2 \bar{c}-(n-1) c_{i}}{(n+1)} \\
& p=\frac{a+c_{i}+(n-1)_{c}^{-}}{(n+1)}
\end{aligned}
$$

En cuanto a los beneficios:

$$
\begin{aligned}
& \Pi_{i}=\left[\frac{a+c_{i}+(n-1)_{c}^{-}}{(n+1)}-c_{i}\right]\left[\frac{a-n c_{i}+(n-1)_{c}^{-}}{b(n+1)}\right] \\
& \Pi_{i}=\left[\frac{a+c_{i}+(n-1)_{c}^{-}-(n+1) c_{i}}{(n+1)}\right]\left[\frac{a-n c_{i}+(n-1)_{c}^{-}}{b(n+1)}\right] \\
& \Pi_{i}=\left[\frac{a-n c_{i}+(n-1) c_{c}^{-}}{(n+1)}\right]\left[\frac{a-n c_{i}+(n-1) c_{c}^{-}}{b(n+1)}\right] \\
& \Pi_{i}=\frac{1}{b}\left[\frac{a-n c_{i}+(n-1)_{c}^{-}}{(n+1)}\right]^{2}
\end{aligned}
$$


Los beneficios de la firma promedio son:

$$
\begin{aligned}
& \Pi_{m}=\left[\frac{a+c_{i}+(n-1) \bar{c}}{(n+1)}-\bar{c}\right]\left[\frac{a-2 \bar{c}+c_{i}}{b(n+1)}\right] \\
& \Pi_{m}=\left[\frac{a+c_{i}+(n-1) \bar{c}-\bar{c}(n+1)}{(n+1)}\right]\left[\frac{a-2 \bar{c}+c_{i}}{b(n+1)}\right] \\
& \Pi_{m}=\frac{1}{b}\left[\frac{a-2 \bar{c}+c_{i}}{(n+1)}\right]^{2}
\end{aligned}
$$

Si $c_{i}=\bar{c}$, se obtienen los resultados tradicionales del modelo de Cour$\operatorname{not}^{32}$.

\section{ANEXO 2}

\section{SISTEMA DE AMORTIZACIÓN CON CUOTAS FIJAS}

Supongamos que se recibe un crédito de valor $X$ en el momento 0 , a una tasa de interés constante $r$, que se cancela en $N$ periodos pagando una suma fija $\theta$ en cada uno:

$X=\frac{\theta}{1+r}+\frac{\theta}{(1+r)^{2}}+\frac{\theta}{(1+r)^{3}}+\ldots+\frac{\theta}{(1+r)^{N}}$

Así, el valor presente de esos pagos debe ser igual a la suma recibida en el momento 0. Factorizando $\mathrm{q} /(1+r)$ se obtiene:

$X=\frac{\theta}{1+r}\left[1+\frac{1}{1+r}+\frac{1}{(1+r)^{2}}+\ldots+\frac{1}{(1+r)^{N}}\right]$

El paréntesis angular es una progresión geométrica, cuya razón es $1 /(1+r)$, de modo que:

$\left[1+\frac{1}{1+r}+\frac{1}{(1+r)^{2}}+\ldots+\frac{1}{(1+r)^{N}}\right]=\frac{\frac{1}{(1+r)^{N}}-1}{\frac{1}{(1+r)}-1}=\frac{\frac{1-(1+r)^{N}}{(1+r)^{N}}}{\frac{1-1-r}{(1+r)}}=\frac{(1+r)\left[1-(1+r)^{N}\right]}{-r(1+r)^{N}}$

Reemplazando, se obtiene:

$\mathrm{X}=\frac{\theta}{(1+\mathrm{r})} \frac{(1+\mathrm{r})\left[1-(1+\mathrm{r})^{\mathrm{N}}\right]}{\left[-\mathrm{r}(1+\mathrm{r})^{\mathrm{N}}\right]}$

Cancelando $(1+r)$ se llega a:

$X=\theta \frac{\left[(1+r)^{N}-1\right]}{\left[r(1+r)^{N}\right]}$

32 Ver, p. ej., Church y Ware (2000, 243-244) o Jehle y Reny (2001, 162).

Revista de Economía Institucional, vol. i9, N.o 37, segundo semestre/2oi7, Pp. 85-ili 4 
Por último, se despeja q para encontrar el valor de la cuota fija.

$\theta=\frac{\left[(1+r)^{N}-1\right]}{\left[(1+r)^{N}\right]}=X \frac{\left[(1+r)^{N}\right]}{\left[(1+r)^{N-1}\right]}$

\section{REFERENCIAS BIBLIOGRÁFICAS}

1. Aghion, P. y Howitt, P. (1999). Endogenous growth theory. Londres: Mit Press.

2. Andonova, V. y Ruiz, G. (2016). The role of industry factors and intangible assets in company performance in Colombia. Journal of Business Research, 69(10), 4377-4384.

3. Arthur Andersen y Co. (1992). The valuation of intangible assets. Reporte especial $\mathrm{P}_{254}$. Londres: Economist Intelligence Unit.

4. Baily, M. y Hall, R. (2002). Intangible assets: Computers and organizational capital. Comments and discussion. Brookings Papers on Economic Activity, 1, 182-198.

5. Ballot, G.; Fakhfakh, F. et al. (2002). Who benefits from training and I\&D: The firm or the workers? A study of panels of French and Swedish firms. ERC working papers in Economics, 2(1), 1-30.

6. Banco Mundial. (2006). Where is the wealth of nations? Measuring capital for the 21 $1^{\text {st }}$ Century. Washington: The World Bank.

7. Becker, B. y Pain, N. (2003). What determines industrial R\&D expenditure in the UK? National Institute of Economic and Social Research, 76(1), 1-34.

8. Becker, G. S. (1983). El capital humano. Un análisis teórico y empirico referido fundamentalmente a la educación [1975]. Madrid: Alianza Editorial.

9. Belkaoui, A. (1992). Accounting theory. Londres: Academy Press.

10. Bergen, D. y Schaaf, S. (2006). Investment spending down again in 2003. Web Magazine, [http://www.cbs.nl/en-gb/menu/themas/macroeconomie-financiele-instellingen/nationale-rekeningen/publicaties/ artikelen/archief/2004/2004-1510-wm.htm].

11. Brynjolfsson, E.; Hitt, L. et al. (2002). Intangible assets: Computers and organizational capital. Brookings Papers on Economic Activity, 1, 137-181.

12. Caballero, R. (1998). Aggregate investment. J. Taylor y M. Woodford (eds.), Handbook of Macroeconomics (pp. 814-862). Amsterdan: North Holland.

13. Cañibano, L.; García, M. et al. (1999). The value relevance and managerial implications of intangibles: A literature review. Proyecto Meritum. 4-57.

14. Chun, H. et al. (2015). Do intangibles contribute to productivity growth in East Asian countries? Evidence from Japan and Korea. RIETI discussion paper $15 \mathrm{E}(055)$.

15. Church, J. y Ware, R. (2000). Industrial organization. A strategic approach. Singapur: McGraw Hill.

16. Coase, R. (1937). The nature of the firm. Economica, 16(4), 386-405. 
17. Coen, R. y Eisner, R. (1987). Investment. J. Eatwell et al. (eds.), The New Palgrave: A dictionary of economics (pp. 981-985). Londres: Macmillan.

18. Corrado, C.; Hulten, C. et al. (2006). Intangible capital and economic growth. NBER working paper, 11948.

19. David, P. (2004). The tale of two traverses. Innovation and accumulation in the first two centuries of US economic growth. Stanford University 05(22), 1-77.

20. Dixit, A. (1979). A model of duopoly suggesting a theory of entry barriers. The Bell Journal of Economics, 10(1), 20-32.

21. Dixit, A. The role of investment in entry-deterrence. The Economic Journal, 90(357), 95-106.

22. Ducharme, L. (1999). Introduction: Main theories and concepts. OECD Measuring intangible investment paper 1.

23. Fukao, K.; Hamagata, S. et al. (2007). Intangible investment in Japan: Measurement and contribution to economic growth. RIETI discussion paper series $07 \mathrm{E}(034)$.

24. Golec, J. y Gupta, N. (2014). Do investments in intangible customer assets affect firm value? Quarterly Review of Economics and Finance, 54(4), 513-520.

25. Hall, R.; Cummins, J. et al. (2000). E-Capital: The link between the stock market and the labor market in the 1990s. Brookings Papers on Economic Activity, 2000(2), 73-118.

26. Hall, R. (2001). The stock market and capital accumulation. American Economic Review, 91(5), 1185-1202.

27. Hennings, K. (1987). Capital as a factor of production. J. Eatwell et al. (eds.), The New Palgrave: A dictionary of economics (pp. 327-332). Londres: Macmillan.

28. Iversen, E. y Kaloudis, A. (2003). IP valuation as a tool to sustain innovation. SINTEF Step Report, 17(03).

29. Jehle, G. y Reny, P. (2001). Advanced microeconomic theory. Boston: Addison Wesley.

30. Johanson, U. (2000). Characteristics of intangibles - Proposals generated from literature and experienced Swedish firms, Proyecto Meritum.

31. Johnson, G. y Scholes, K. (2001). Dirección estratégica. Madrid: Prentice Hall.

32. Lev, B. (2003). Remarks on the measurement, valuation and reporting on intangible assets. Federal Reserve Bank of New York Economic Policy Review, 9(3), 17-22.

33. Lorente, J. (2000). Inversión en intangibles y creación de valor en la industria manufacturera española. Economía Industrial, (332), 109-123.

34. Lynch, L. y Black, S. (1995). Beyond the incidence of training: Evidence from a national employer's survey. National Bureau of Economic Research, (5231), 2-32.

35. Maitlan, J. conde de Lauderdale. (1804). An inquiry into the nature and origin of public wealth. Londres: Archibald Constable \& Co.

36. Mankiw, G. (2006). Intangible investment. [http://gregmankiw.blogspot. com/2006_04_01_archive.html]. 
37. McGrattan, E. y Prescott, E. (2010). Unmeasured investment and the 1990's us hour boom. Federal Reserve Bank of Minneapolis, (369), 1-49.

38. Mincer, J. (1989). Human capital. Responses to technological change in the labour market. NBER working paper 3207.

39. Nakamura, L. (1999). Intangibles: What put the new in the new economy? Business Review. Federal Reserve Bank of Philadelphia, julio-agosto, 3-16.

40. Nakamura L. (2001). What is the us gross investment in intangibles? (At least) one trillion dollars a year! Federal Reserve Bank of Philadelphia, 01(15), 2-41.

41. Nicholson, W. (1972). Microeconomic theory. Basic principles and extensions. Chicago: Dryden Press.

42. Nicholson, W. (2001). Microeconomía intermedia y sus aplicaciones. Bogotá: McGraw Hill.

43. OCDE. (1999). National efforts to measure intangible investment. OECD Measuring intangible investment paper 5.

44. OCDE. (2012). Corporate reporting of intangible assets: A progress report. [https://www.oecd.org/daf/ca/Intangible\%20Assets.pdf].

45. O'Mahony, M. y Vecchi, M. (2002). Do intangible investments affect companies' productivity performance? National Institute of Economic and Social Research, Londres.

46. Peneder, M. (2002). Intangible investment and human resources. Journal of Evolutionary Economics, 12(1-2), 107-134.

47. Porter, M. (1986). Estrategia competitiva. México DF: Compañía Editorial Continental.

48. Robinson, W. y Chiang, J. (1996). Are Sutton predictions robust? Empirical insights into advertising, R\&D and concentration. Journal of Industrial Economics, 4(44), 389-408.

49. Rodríguez C., A. y Araújo de la M., A. (2002). Algunas consideraciones en la valoración del capital intelectual. L. Batz y F. Cossio (eds.), The limits of the firm (pp. 427-435). Paris.

50. Romer, P. (1990). Endogenous technological change. Journal of Political Economy, 98(5), 71-102.

51. Romer, D. (2001). Advanced macroeconomics. Nueva York: McGraw Hill.

52. Sachs, J. y Larraín, F. (1994). Macroeconomía en la economía global. México DF: Prentice Hall.

53. Shy, O. (1996). Industrial organization. Theory and applications. Cambridge: Mit Press.

54. Singh, N. y Vives, X. (1984). Price and quantity competition in a differentiated duopoly. RAND Journal of Economics, 4(15), 546-554.

55. Slywotzky, A.; Wise, R. y Weber, K. (2004). Cómo crecer cuando los mercados no crecen. Bogotá: Norma.

56. Sutton, J. (1996). Sunk costs and market structure. Cambridge: Miт Press.

57. Tasi, L. y Yen, D. (2012). Determinants of intangible assets value: The data mining approach. Knowledge-Based Systems, 31, 66-77.

58. Tirole, J. (2002). The theory of industrial organization. Cambridge: Mit Press.

59. Uchitelle, L. (2006). Seizing intangibles for the GDP. New York Times [http://www.nytimes.com/2006/04/09/business/yourmoney/09view. html?_r=2\&oref=slogin\&oref=slogin]. 
60. Varian, H. (1992). Análisis microeconómico. Barcelona: Antoni Bosch. 61. Veblen, T. (1908). On the nature of capital: Investment, intangible assets, and the pecuniary magnate. Quarterly Journal of Economics, 23(1), 104-136.

62. Vosselman, W. (1999). Initial guidelines for the collection and comparison of data on intangible investment. OECD Measuring intangible investment paper 4.

63. Webster, E. (1999). The growth of enterprise intangible investment. Melbourne Institute of Applied Economic and Social Research, working paper 9.

64. Webster, E. (2002). Intangible and intellectual capital: A review of the literature. Melbourne Institute of Applied Economic and Social Research, working paper 10.

65. Webster, E. (2006). Investment in intangible capital: An enterprise perspective. The Economic Record, 82(256), 82-96.

66. Young. A. (1999). Towards an interim statistical framework: Selecting the core components of intangible investment. OECD Measuring intangible investment paper 3 . 